\title{
Modelo de comunicación autista desde los lineamientos metodológicos de la teoría cognitiva sistémica de la comunicación
}

\section{Autistic communication model from the methodological guidelines of the communication systemic cognitive theory}

Marilia L. Baquerizo Sedano'

Universidad Continental

\section{RESUMEN}

Objetivos: Construir un modelo que explique el fenómeno de comunicación autista desde los lineamientos metodológicos de la teoría cognitiva sistémica de la comunicación. Métodos: El trabajo es de enfoque mixto, diseño transformativo secuencial, nivel descriptivo y tipo básico. Se recogieron datos cuantitativos ( $n=30, D x .[299,00]$, [F84,0]) con el Children's Communication Cheklist-2, versión de investigación, un instrumento que considera los aspectos formales y pragmáticos del lenguaje, se cualificaron esos datos y se construyó sobre ellos un modelo mediante un proceso cualitativo de teoría fundamentada, relacionando primero las características a nivel biológico y físico del autismo que pueden explicar los datos obtenidos, y luego las características a nivel psicológico y metafísico que pueden mejorar la comprensión global del sistema. Resultados: En los niños autistas verbales no existe una estricta dicotomía entre las dificultades en los aspectos formales y pragmáticos del lenguaje, y no es posible determinar cuáles son las alteraciones lingüísticas propias del fenómeno de comunicación autista. La base genética y nerviosa del autismo es heterogénea y compleja. Variaciones genéticas parecen condicionar el desarrollo nervioso embrionario y temprano, lo que conlleva a diferencias cerebrales estructurales y funcionales, sustentadas en alteraciones neuroquímicas. Conclusiones: La construcción del modelo se ha limitado considerablemente por la ambigüedad de los datos cuantitativos y cualitativos, a pesar de ello, es posible vincular las resultados de cada etapa y

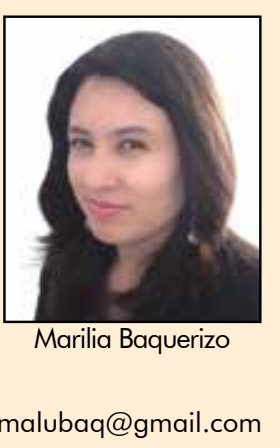

sugerir que a un cerebro diferente (a causa de la heterogeneidad genética y la complejidad nerviosa) le corresponde un estilo cognitivo diferente (producto de una percepción, memoria y pensamiento particular); de ellos, emergen nuevas formas de comunicación, que desde la mirada de la neurodiversidad, pasan de ser déficits a ser elementos funcionales.

Palabras clave: Autismo, comunicación, cognitivo-sistémico, neurodiversidad. 


\section{ABSTRACT}

Objectives: To constructa model that explains the autistic communication phenomenon model from the methodological guidelines of the communication systemic cognitive theory. Methods: The work is a mixed approach, sequential transformative design, descriptive level and basic type. Quantitative data ( $\mathrm{n}=30$, Dx. $[299,00]$, [F84,0]) were collected with the Children's Communication Cheklist-2, research version, an instrument that considers the formal and pragmatic language aspects, such data were skilled and upon them a model was built through a grounded theory qualitative process. First, relating the features at the autism biological and physical level that can explain the obtained data, and then the features at the psychological and metaphysical level that can improve the system overall understanding. Results: In the verbal autistic children don't exist a strict dichotomy between the difficulties in language formal and pragmatic aspects and it is not possible to determine which linguistic alterations from the autistic communication phenomenon are. The autism genetic and nervous basis is heterogeneous and complex. The genetic variations seem to determine the embryonic and early nervous development, which leads to structural and functional cerebral differences sustained in neurochemical alterations. Conclusions: The model construction has been considerably limited by the quantitative and qualitative data ambiguity. Nevertheless, it is possible to link the results of each stage and suggest that: to a different brain (due to genetic heterogeneity and nervous complexity) it's corresponded a different cognitive style (result of the perception, memory and particular thought); of which emerge new communication ways. From the look of the neurodiversity, go from being deficits to be functional elements.

Keywords: Autism, communication, cognitive-systemic, neurodiversity.
INTRODUCCIÓN

Los trastornos de espectro autista (TEA) son trastornos del desarrollo de inicio precoz, que comportan alteraciones en la interacción social, la comunicación, y la flexibilidad de conductas, intereses y actividades (1). Hace tres décadas, se estimaba que una de cada diez mil personas en el mundo, cumplía con los criterios de algún TEA (2); investigaciones recientes en diferentes países señalan que actualmente es una de cada ochenta (3). Este alarmante incremento se ha intentado explicar desde los posibles factores contribuyentes; sin embargo, no está claro aún si es producto de cambios en las definiciones clínicas que actúan como base de los estudios epidemiológicos o se trata de un aumento real debido a variables ambientales (4).

Los cambios en las definiciones clínicas se dan como consecuencias temporales de discusiones nosográficas, y se han dado desde que Eugen Bleuler en 1911 introdujera por primera vez el término autismo (5). La compleja naturaleza de los TEA y la ausencia de marcadores biológicos para el diagnóstico, hacen que aunque los síntomas sean evidentes y suelan presentarse antes de los tres años (3), los TEA sigan siendo vistos con un halo de extrañeza e incapacidad.

Los sistemas de clasificación y diagnóstico, especialmente desde hace tres décadas (DSM III-IV-IV.TR; CIE10), han intentado ordenar los conocimientos con el fin de capturar científicamente el espectro. En el Manual Diagnóstico y Estadístico de los Transtornos Mentales (DSM) no se contempla el concepto de espectro y se incluye los TEA dentro de los trastornos generalizados del desarrollo (TGD). En esta categoría, se diferencia al trastorno autista, trastorno de Rett, trastorno desintegrativo infantil, trastorno de Asperger y trastorno generalizado del desarrollo no especificado de otra forma (6).

El trastorno autista $[299,00]$, recogido en la CIE 10 con el nombre de autismo infantil 
[F 84,0], es considerado la patología de comunicación por antonomasia (7), y en la práctica psiquiátrica, la presencia o ausencia de deficiencias comunicativas es fundamental, pues a partir de ellas se infiere alteraciones en la interacción social y la flexibilidad comportamental.

Sin embargo, las deficiencias comunicativas para la práctica psiquiátrica son exclusivamente trastornos de lenguaje. Cuando Riviere y Belinchon en 1981 (8), asentaron las bases para conceptuar el autismo, reconocieron a este como una tendencia natural de evitar el uso del lenguaje como instrumento de comunicación y representación. $Y$ a partir de ellos se considera que no hay funciones de interacción comunicativa que no pasan por el lenguaje representacional (7).

López (9), después de realizar una revisión crítica del discurso actual de la psiquiatría y psicología, encuentra que esta concepción se corresponde con el modelo lingüístico estructuralista de Saussure y Chomsky, que considera que no hay funciones de interacción comunicativa que no pasan por el lenguaje representacional. Enuncia, cuestionando a esta concepción, que más que incapacidad o alteración en la comunicación, el autismo plantea la emergencia de nuevas lógicas delirantes propias, a partir de las cuales se procura una nueva salud.

La apuesta teórico-crítica que presenta López (7), da cuenta del cariz positivo y productivo de los TEA. Se infiere a partir de ella, que por su naturaleza, los TEA tienen dos compuestos, uno objetivo y otro subjetivo. Lo objetivo es la parte que existe independientemente del observador, lo que se consideraría irrefutable y se relaciona con la base física y biológica. Lo subjetivo, es más bien lo ambiguo y relativo, la parte dependiente del observador, sobre las que se dan interminables discusiones nosográficas, que correspondería a niveles psicológicos y metafísicos.

\section{MATERIAL Y MÉTODOS}

Con el fin de demostrar la validez de la apuesta teórica de López y mejorar la comprensión del fenómeno de comunicación autista, se intentó construir un modelo, asumiendo como base los componentes objetivos y replanteando los subjetivos. El modelo fue construido sobre la base de los lineamientos metodológicos de la teoría cognitiva sistémica de la comunicación (10), que desde una mirada integral, precisamente, configura la comunicación en los niveles físico, biológico, psicológico y metafísico.

Por tratarse el fenómeno de la comunicación del trastorno autista, un campo altamente complejo, se planteó abordarlo de forma sistemática, desde un enfoque mixto y un diseño transformativo secuencial que incluyó la realización de tres etapas. La primera corresponde a un proceso cuantitativo y los dos siguientes a un proceso cualitativo.

En la etapa I se recogió datos cuantitativos $(n=30$, Dx. $[299,00],[F 84,0])$, utilizando el Children's Communication Cheklist-2, versión de investigación (11), un instrumento que considera los aspectos formales (fonología, sintaxis, semántica, coherencia) y pragmáticos (inicio inapropiado, lenguaje estereotipado, uso del contexto, comunicación no verbal) de la comunicación. El instrumento proporciona además dos puntaciones compuestas, el compuesto comunicativo general (GCC), que permite discriminar entre niños con problemas comunicativos $(\leq 54)$ y niños con desarrollo típico; y el compuesto de problemas de interacción social (SIDC), que permite identificar a aquellos niños con dificultades pragmáticas y sociales desproporcionadas en relación con su discapacidad en el lenguaje formal $o$ estructural.

Las dos siguientes etapas de la investigación evalúan de qué manera se integran o adquieren sentido los conocimientos en el nivel biológico, físico, psicológico y metafísico. Para ello fue necesario revisar 
las teorías y ubicar las características del autismo que puedan explicar la comunicación autista (desde lo que plantean los enfoques tradicionales y proponen los enfoques alternativos).

Después de cualificar los datos, en la etapa II se recolectó información secundaria sobre las características a nivel biológico y físico del autismo utilizando una bitácora de investigaciones recientes. Se construyó sobre ellos un modelo, mediante un proceso cualitativo de teoría fundamentada, describiendo primero cómo se investiga el autismo en este nivel, organizando los conocimientos sobre los hallazgos de laboratorio genéticos y del sistema nervioso (de forma general y específicamente su correlato de la disfunción lingüística, que según las teorías contemporáneas lo caracterizan), para luego analizar la correspondencia de los hallazgos de laboratorio con los resultados de la primera etapa. Después de ello, se evaluó específicamente una posible correspondencia entre nuestras inferencias y los hallazgos en las dos áreas, para finalmente integrar (en un esquema explicativo) los hallazgos de laboratorio a este nivel y establecer su posible relación con la propuesta crítica de López.

En la etapa III se recolectó información secundaria sobre las características a nivel psicológico y metafísico del autismo utilizando los mismos intrumentos de la segunda etapa y considerando los aspectos de ingreso de la información: percepción, conservación de la información (memoria), procesamiento de la información (pensamiento) y una mirada evolutiva. También se construyó un modelo y se hizo una correspondencia de los hallazgos con los resultados de la primera etapa.
Tabla $N^{\circ}$ 1: Medias y desviaciones estándar de los puntajes compuestos

\begin{tabular}{crrrr}
\hline Puntaje compuesto & M & SD & MIN & MAX \\
\hline GCC & 29,07 & 13,94 & 5 & 60 \\
SIDC & 3,57 & 7,23 & -23 & 18
\end{tabular}

Finalmente, se vinculó las conclusiones de cada etapa y se generó un modelo explicativo.

\section{RESULTADOS}

Etapa I. Aspectos formales y pragmáticos del lenguaje propios del fenómeno de comunicación autista.

Tabla № 2: Medias y desviaciones estándar de los puntajes índice

\begin{tabular}{lcccc}
\hline Puntaje compuesto & M & SD & MIN & MAX \\
\hline Aspectos formales & 13,23 & 9,35 & 0 & 41 \\
Aspectos pragmáticos & 15,33 & 6,75 & 5 & 29 \\
Aspectos de & & & & \\
comunicación social & 8,20 & 4,05 & 1 & 15 \\
\hline
\end{tabular}

Los estadísticos descriptivos del compuesto comunicativo general (GCC) y del compuesto de problemas de interacción social (SIDC), indican que globalmente los problemas comunicativos son significativos ( $\mathrm{mGCC}=$ $29,07)$ y, el desarrollo lingüístico del grupo es armónico o típico (mSIDC=3,57). (tabla $\left.\mathrm{N}^{\circ} 1\right)$

Al analizar individualmente cada GCC, se observa que un niño no tiene problemas

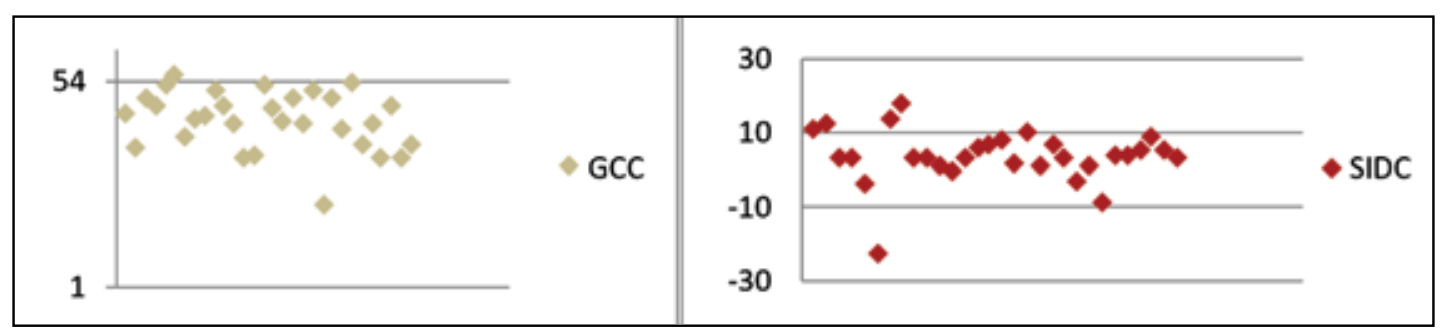

Figura № 1: Dispersión de GCC y SIDC muestrales (eje x: puntaje obtenido; eje y: sujetos muestrales) 
comunicativos significativos: 29 niños están bajo la línea $(\leq 54)$ y uno está sobre ella $(>54)$.

Y al analizar individualmente cada SIDC, se observa que de los 30 casos muestrales, en 4 el SIDC es $\geq 11$ (puntaje usual en TEL), en 25 el SIDC está entre - 10 y 10 (puntaje típico) y en 1 el SIDC es $\leq-11$ (puntaje usual en TEA).

Con los estadísticos descriptivos de los puntajes índice (tabla $\mathrm{N}^{\circ} 2$ ), damos cuenta las dificultades formales en manera global, son ligeramente mayores que las dificultades pragmáticas.

Para comprobar que esta ligera diferencia no es significativa, se hallaron las y pragmáticos no están polarizadas sino mezcladas por orden de rendimiento.

En general, la escala de sintaxis es la que presenta el más bajo rendimiento, mientras que la de inicio inapropiado el mejor.

Dentro de los aspectos formales, la escala en la que la muestra presenta más problemas es la de sintaxis, seguida por el habla, la semántica y la coherencia. Dentro de los aspectos pragmáticos, la escala en la que la muestra presenta más problemas es la de comunicación verbal, seguida por el uso del contexto, lenguaje estereotipado e inicio inapropiado.

El 96,7\% de los niños que componen la muestra tienen problemas comunicativos

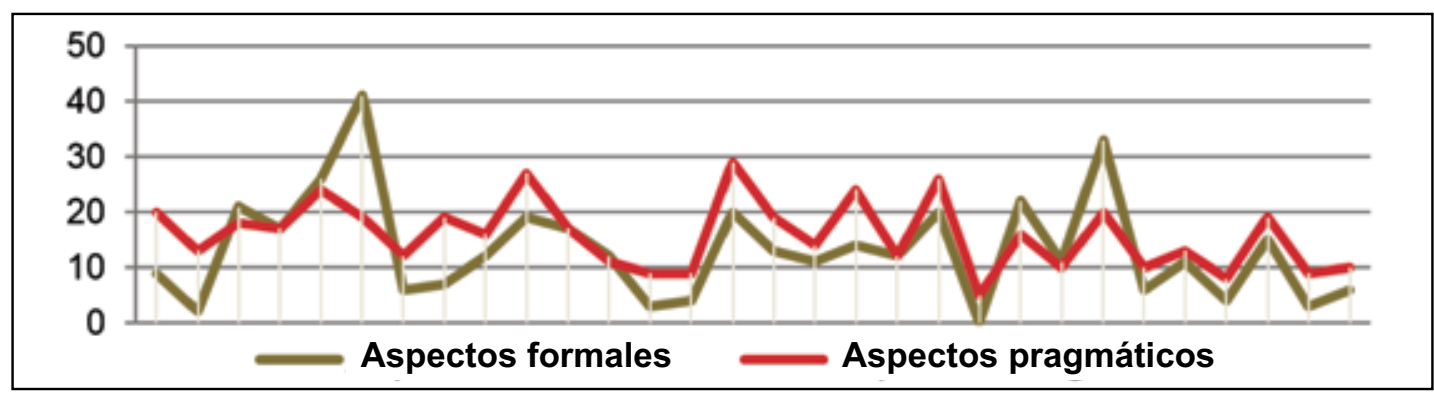

Figura $\mathrm{N}^{\circ}$ 2: Comparación entre puntajes índice pragmáticos y formales

correlaciones de Pearson entre los puntajes índice. Se encontró que entre el índice pragmático y formal $(0,608)$, y entre el índice de comunicación social y formal $(0,578)$, la asociación es positiva media; y entre el índice de comunicación social y pragmático $(0,779)$, la asociación es positiva considerable.

Al analizar individualmente las diferencias entre los puntajes índice de los aspectos pragmáticos y formales (figura $\mathrm{N}^{\circ} 2$ ), se observa que en 20 casos las dificultades son más formales que pragmáticas, en 3 casos las dificultades formales son tan igual que las pragmáticas y en 7 casos las dificultades son más pragmáticas que formales.

Los puntajes escalares ordenados de forma descendente respecto a la media (tabla $\mathrm{N}^{\circ} 3$ ), indican que existe un déficit significativo en todas las escalas y que las que corresponden a los aspectos formales significativos. Los perfiles lingüísticos (PL) no corresponden a lo esperado desde la teoría que sustenta la prueba que diferencia tres perfiles en función de las dificultades pragmáticas, formales y de

Tabla №3: Medias y desviaciones estándar de los puntajes escalares *

\begin{tabular}{lrrrr}
\hline & & & & \\
\hline Escala & $M$ & SD & MIN & MAX \\
\hline E Inicio inapropiado & 5,70 & 2,22 & 2 & 10 \\
J Intereses & 5,43 & 2,37 & 1 & 11 \\
D Coherencia & 4,23 & 2,6 & 0 & 13 \\
C Semántica & 3,93 & 2,36 & 0 & 9 \\
F Lenguaje & 3,93 & 2,43 & 0 & 11 \\
$\quad$ estereotipado & & & & \\
G Uso del contexto & 3,30 & 1,86 & 0 & 7 \\
A Habla & 3,20 & 3,31 & 0 & 12 \\
H Comunicación no & 2,90 & 1,63 & 0 & 6 \\
$\quad$ verbal & & & & \\
I Relaciones sociales & 2,77 & 2,69 & 0 & 9 \\
B Sintaxis & 1,87 & 2,83 & 0 & 12 \\
\hline * A mayor puntaje, mejor rendimiento & & &
\end{tabular}


comunicación social. Según la teoría, en el trastorno especifico del lenguaje (TEL) se da considerables dificultades formales y escasas o nulas dificultades pragmáticas y de comunicación social; en el TEA, en cambio, se da considerables dificultades pragmáticas y de comunicación social y escasas o nulas dificultades formales (disminuidas aún más en el Asperger); y en el perfil armónico o típico, se da un rendimiento sin diferencias significativas entre estos tres aspectos.

Tampoco, los puntajes escalares y su mezcla en orden de rendimiento corresponden a lo esperado, pues en el TEA, las escalas pragmáticas deben ubicarse en su mayoría debajo de las escalas formales. Porque no están polarizadas, no se puede determinar nivelen entre sí. Por otro lado, la muestra es pequeña, estuvo conformada por autistas verbales y el promedio de edad es de 10,01 $(S D=2,67)$, aunque se haya probado que la edad no es una variable que interfiere, es necesario una muestra más amplia y un rango más amplio de edad para evaluar si el desarrollo lingüístico en el autismo sigue un ritmo propio, pues tal vez el perfil lingüístico asociado clásicamente al autismo, no sea fijo sino cambiante en función de la edad.

Dejando a un lado las posibles variables intervinientes, los resultados permiten reflexionar sobre la naturaleza lingüística de los TEA, en específico del autismo, y pueden sugerir la presencia de varios perfiles lingüísticos en él o un solapamiento con otros trastornos del lenguaje, particularmente con

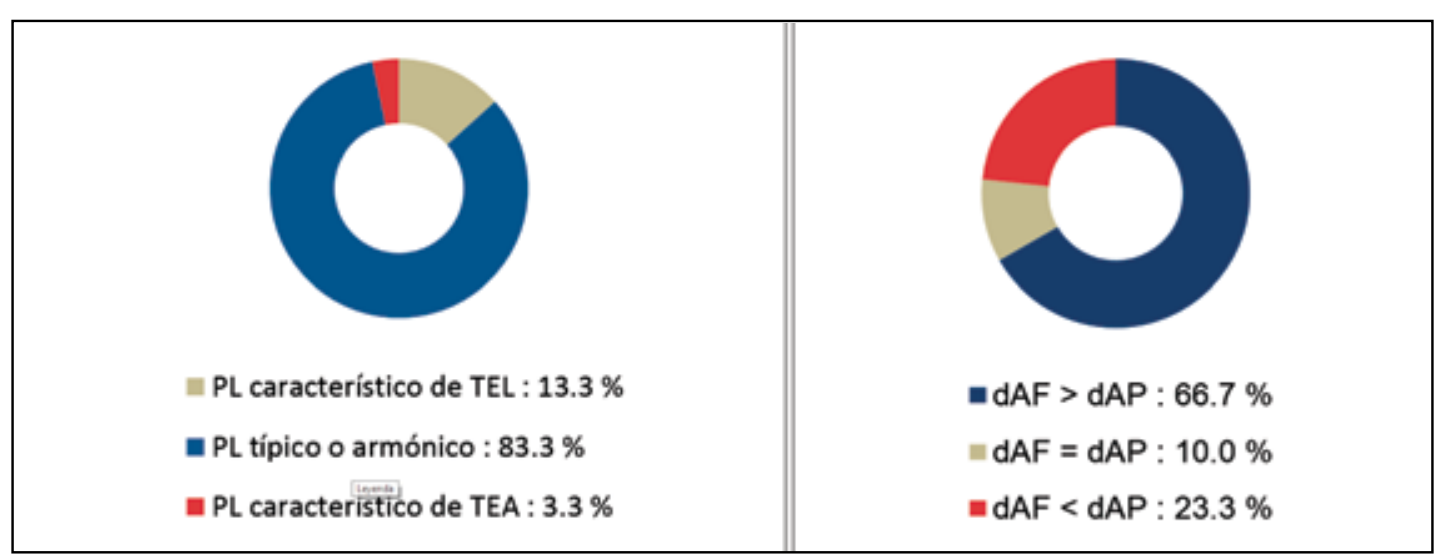

Figura $N^{\circ}$ 3: Perfiles lingüísticos en la muestra y comparación de dificultades formales y pragmáticas

cuáles son las alteraciones lingüísticas formales o pragmáticas propias del fenómeno de comunicación autista.

Encontrar que en la muestra el PL típico o armónico sea el más común (figura $\mathrm{N}^{\circ}$ 2) y que las dificultades en los aspectos pragmáticos en la mayoría de casos sean menores que las dificultades en los aspectos formales (figura $N^{\circ} 3$ ), lleva primero a buscar características muestrales específicas que puedan haberse convertido en variables intervinientes. El grupo muestral está compuesto por niños que llevan asistiendo 2 o más años a un colegio especializado que usa un modelo educativo enfocado en el desarrollo de la comunicación pragmática antes que en la comunicación formal, quizás ello hizo que ambos aspectos se el $T E L$, que tradicionalmente se considera como claramente distinto, pero que según algunas investigaciones puede tener muchas semejanzas con el autismo, a nivel fenotípico y etiológico (12).

La idea de que existen varios perfiles lingüísticos en el autismo ha sido trabajada por Rapin y Dunn (13). Ellos diferencian dos subtipos de perfiles en función de sus capacidades (o dificultades) lingüísticas. El primero estaría conformado por quienes presentan un desarrollo normal en lo formal con dificultades en lo pragmático, y el segundo por quienes manifiestan un problema tanto en lo formal como en lo pragmático (14). La proporción entre las poblaciones que pertenecerían a ambos subtipos sería 2:1 ○ 3:1 (15). Siguiendo 
esta tipología, la proporción en la muestra sería 1:29, lo cual no iría en contra de la hipótesis por tratarse de una muestra pequeña y no representativa.

A nivel fenotípico y etiológico (genética y neuroanatómicamente), el segundo subtipo es el que guardaría más semejanza con el TEL. Los niños autistas verbales presentan más dificultades formales de lo que se pensaba $(14,16)$ y los niños con $\mathrm{TEL}$, presentan además de problemas pragmáticos secundarios (a consecuencia de las dificultades formales), problemas pragmáticos primarios (per se) $(17,18)$. Las coincidencias etiológicas son resaltantes, la región cromosómica 7q31 alberga dos de los loci asociados al TEL y al locus AUTS1B, relacionado con las anomalías lingüísticas graves en el autismo (19); las regiones cerebrales asociadas al procesamiento del lenguaje como el área de Broca suele ser menor de lo habitual tanto en los individuos con TEL como en los que pertenecen al segundo subtipo de autistas, también se ha comprobado que el área temporal es mucho más asimétrica de lo normal en ambos grupos.

La diferencia clara entre el autismo y el TEL, es que el autismo (trastorno generalizado) afecta a tres dimensiones (lingüística, social y conductual), mientras que el TEL (trastorno específico) se restringe a la dimensión lingüística. No está claro aún si los paralelismos son consecuencia de un déficit subyacente común a ambos trastornos - dos déficits subyacentes distintos dan lugar a un fenotipo cognitivo y lingüístico semejante (16).

De cualquier forma, sobre la base de los resultados cuantitativos obtenidos y las investigaciones recientes relacionadas, podemos concluir que en el funcionamiento lingüístico de los niños autistas verbales que conformaron la muestra no existe una estricta dicotomía entre las dificultades en los aspectos formales y pragmáticos del lenguaje, y no es posible determinar cuáles son las alteraciones lingüísticas propias del fenómeno de comunicación autista. La muestra presenta más dificultades en los aspectos formales de lo que se esperaba, indicando así la posible presencia de varios perfiles lingüísticos en el autismo y sugiriendo un solapamiento entre el autismo y otros trastornos de lenguaje.

Etapa II. Características a nivel biológico y físico del autismo que pueden explicar los aspectos formales y pragmáticos del lenguaje propios del fenómeno de comunicación autista.

El autismo tiene un porcentaje alto de heredabilidad, pero presenta una heterogeneidad genética extrema. El correlato genético (cromosomas y loci) de la disfunción lingüística en el autismo (particularmente, la región cromosómica 7q31), plantea, como anteriormente se había comentado, la posibilidad de que existan determinantes genéticos comunes con otros trastornos de lenguaje, en particular con el TEL. Lo que explicaría las semejanzas que se advierten entre ellos a nivel fenotípico.

El condicionamiento genético al parecer se expresa en la etapa embrionaria o temprana, lo que lleva a una condición cerebral diferente a nivel estructural y funcional. Las alteraciones lingüísticas no tienen una correspondencia clara con alguna neuropatología, las áreas y circuitos cerebrales implicados en el lenguaje (también asociadas a la memoria de trabajo y teoría de la mente) sí presentan alteraciones. La teoría de conectividad permite comprender sistémicamente algunos elementos conductuales, sin embargo, solo las investigaciones sobre los procesos neuroquímicos explicarían la forma diferente en que funciona el cerebro autista.

Los endofenotipos lingüísticos desde los que se investiga a nivel biológico y físico, no consideran la dicotomía en los aspectos formales y pragmáticos del lenguaje. Éstos son altamente específicos, pues es necesario cuantificarlos y aislarlos (en la medida de lo posible). En vista de que la razón entre las dificultades en los aspectos formales y pragmáticos no logran, como habíamos 
analizado en la primera etapa, captar realmente el fenómeno de comunicación autista, quizás no es necesario considerarlos tampoco a nivel fenotípico, dentro de los criterios diagnósticos.

En torno a lo analizado y señalado en el esquema, podemos concluir en esta etapa que la base genética y nerviosa del autismo es heterogénea y compleja. Variaciones genéticas parecen condicionar el desarrollo nervioso embrionario y temprano, lo que conlleva diferencias cerebrales estructurales y funcionales, sustentadas en alteraciones neuroquímicas.

Las características a nivel biológico y físico del autismo, especialmente relacionadas con la comunicación, son estudiadas desde endofenotipos lingüísticos específicos que no diferencian los aspectos formales y pragmáticos. Los hallazgos no establecen relaciones causales claras y demuestran la superposición también en este nivel del autismo con otros trastornos de lenguaje y otros trastornos más generales, como la esquizofrenia y la depresión.

Etapa III. Características a nivel psicológico y metafísico del autismo que pueden explicar los aspectos formales y pragmáticos del lenguaje propios del fenómeno de comunicación autista.

La heterogeneidad genética y la complejidad nerviosa condicionan las características a nivel psicológico (cognitivo). Diversas investigaciones sugieren que las alteraciones cognitivas y lingüísticas están altamente relacionados con el autismo, pero la naturaleza de esta asociación y el papel que representan las dificultades lingüísticas no están completamente clarificados.

En este nivel han surgido grandes teorías que explican el fenotipo autista: teoría de la coherencia central débil, teoría de la disfunción ejecutiva, teoría de la ceguera mentaly teoría de la empatía-sistematización. Analizaremos el ingreso, conservación y procesamiento de información tomando en cuenta y adaptando los planteamientos de estas teorías.
Varias teorías permiten explicar las alteraciones lingüísticas en el autismo, la teoría de la ceguera mental y la teoría de la empatía-sistematización parecen ser la más efectivas. Estas teorías tiene un carácter integrativo, que logra conectar (aunque todavía no directamente) los hallazgos neurobiológicos y las conductas en cada dimensión afectada, por ello actualmente son las de mayor valor.

Ambas teorías explican tanto los aspectos formales como pragmáticos del lenguaje autista, pero especialmente alcanzan a atribuir causas al déficit pragmático. Por otro lado, éstas teorías y los conocimientos alcanzados sobre el ingreso, conservación y procesamiento de la información en el autismo, sugieren que se configura un estilo cognitivo particular y diferente.

Es probable que sobre este estilo, se generen otras formas de comunicación también particulares y diferentes, que pueden ser alteraciones en los aspectos formales y pragmáticos del lenguaje (desde un enfoque clásico y psiquiátrico), pero pueden ser también expresiones auténticas (desde un enfoque alternativo).

Sobre la base de lo analizado y señalado en el esquema, podemos concluir en esta etapa que las características a nivel psicológico del autismo sugieren particularidades en el ingreso (percepción), conservación (memoria) y procesamiento de la información (pensamiento).

La comunicación autista, por ello, puede verse como consecuencia de un estilo cognitivo, que al parecer tiende a la sistematización y se aleja de la empatía. Este estilo cognitivo diferente se relaciona con las diferencias cerebrales, y en conjunto constituyen una diferencia humana normal, de valor evolutivo.

\section{DISCUSIÓN}

El fenómeno de comunicación autista es 


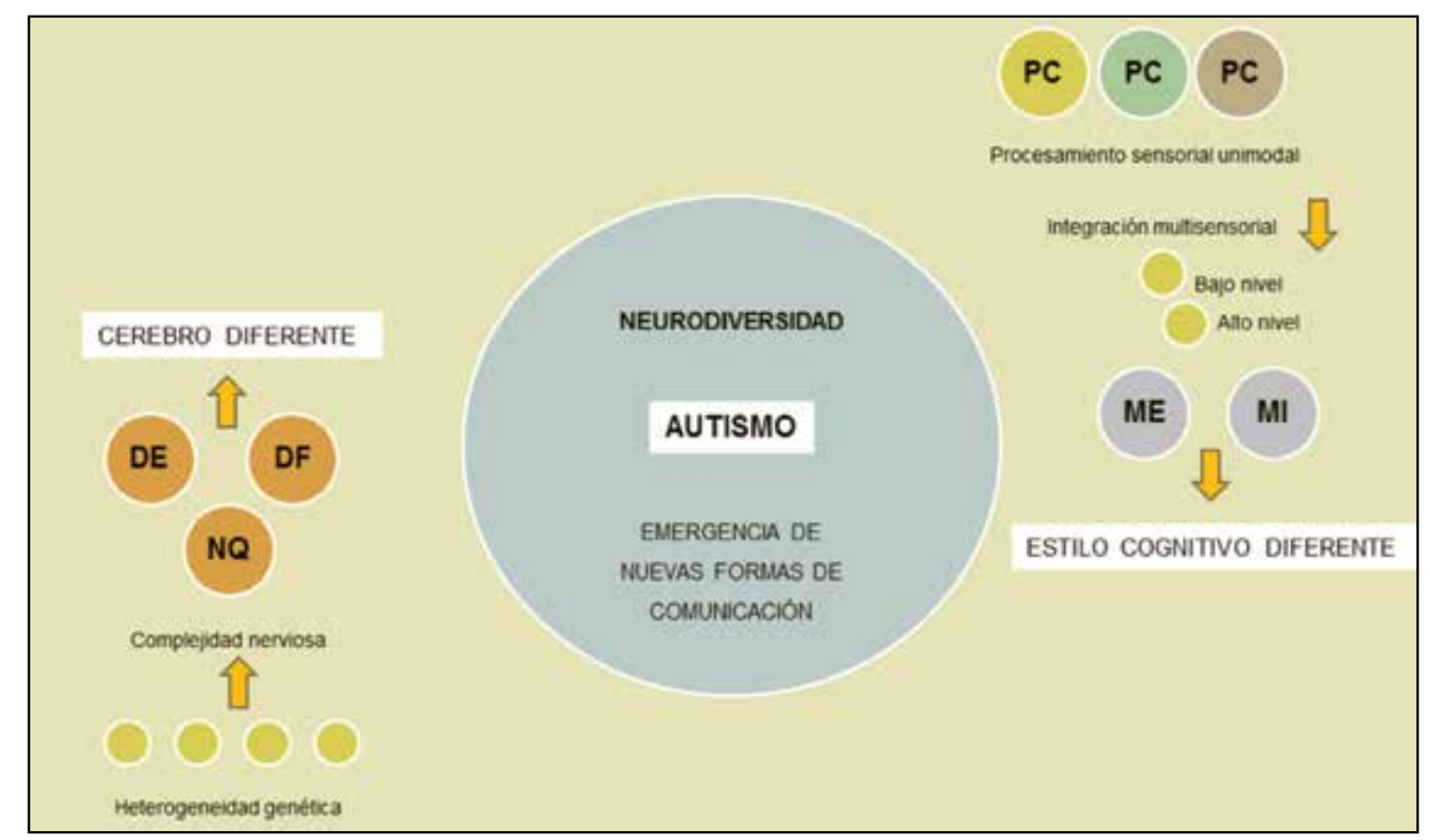

Figura $\mathrm{N}^{\circ}$ 4: Modelo explicativo del fenómeno de comunicación autista.

sumamente complejo, los lineamientos metodológicos de la teoría cognitiva sistémica de la comunicación han clarificado nuestra visión de la tipología y etiología autista y han permitido organizar los avances sobre las características a nivel biológico, físico, psicológico y metafísico que podrían explicar los aspectos formales y pragmáticos del lenguaje; pero la construcción del modelo se ha limitado considerablemente por la ambigüedad de los datos cuantitativos y cualitativos.

Es posible vincular las conclusiones de las tres etapas. A un cerebro diferente (a causa de la heterogeneidad genética y la complejidad nerviosa) le corresponde un estilo cognitivo diferente (producto de una percepción, memoria y pensamiento particular). De ellos emergen nuevas formas de comunicación que desde la mirada de la neurodiversidad, pasan de ser déficits a ser elementos funcionales.

El autismo está definido por su afección a tres dimensiones. Como habíamos planteado en el análisis de los resultados cuantitativos, la lingüística clásica ejerce poder sobre ellas, sobretodo en la dimensión de comunicación, pues configura alteraciones en función de los aspectos formales y pragmáticos del lenguaje, y diferencia (ineficazmente) al grupo diagnóstico de otros grupos clínicos en razón de estas alteraciones.

Con los resultados cuantitativos (etapa I) se valida de alguna manera la apuesta teórico-crítica de López, nuestro principal antecedente, pues la lingüística impone coordenadas semióticas sobre el diagnóstico, pero ellas no logran aprehender al amplio fenotipo autista.

Para evaluar si las supuestas alteraciones en los aspectos formales y pragmáticos se corresponderían con características objetivas (nivel físico y biológico) y subjetivas (nivel psicológico y metafísico), se revisó el estado de conocimiento sobre el autismo y especificamente, su componente lingúístico. El resultado cualitativo (etapa II y III) puso de manifiesto que también en estos niveles no se logra aprehender al autismo y solo se cuenta con aproximaciones causales que dan cuenta de una enorme complejidad y heterogeneidad.

Los lineamientos metodológicos de la teoría cognitiva sistémica de la comunicación han permitido organizar los conocimientos sobre la tipología y etiología autista. Se ha alcanzado a explorar el fenómeno 
de comunicación autista, y aunque no se ha logrado (como se pretendía) construir un modelo explicativo, hemos puesto en frente la necesidad de recomprender su naturaleza. Este modelo como sugiere la conclusión final, ha sido limitado por la ambigüedad de los datos y las relaciones de manera general entre los niveles. Sin embargo, al vincular los resultados en las tres etapas, se planteó que de un cerebro diferente y un estílo cognitivo particular emergen nuevas formas de comunicación, pueden verse como déficit o carencia (desde el enfoque tradicional), pero también como lógicas delirantes no patológicas y productivas (desde el enfoque alternativo, de López, y la neurodiversidad).

\section{REFERENCIAS BIBLIOGRÁFICAS}

1. Artigas J. El lenguaje en los trastornos autistas. REV NEUROL. 1999; 28(2): $118-123$.

2. Molina S. Detección temprana de autismo żes posible y necesaria? Revista CES Psicología. 2012; 5(1): 112-117.

3. Baio J. Prevalence of Autism Spectrum Disorders - Autism and Developmental Disabilities Monitoring Network, 14 Sites, United States, 2008. MMWR. 2008; 61(3): 1-19.

4. Charman T. The Highs and Lows of Counting Autism. Am J Psychiatry. 2011; 168: 873-875.

5. Garrabé J. El autismo. Historia y clasificaciones. Salud Mental. 2012; 35(3): 257-261.

6. American Psychiatric Association. Diagnostic and statistical manual of mental disorders. Washington, DC: American Psychiatric Association; (2000; 2002). 4ta. ed.

7. López J. Atravesando una muralla invisible: Teorías de la comunicación y semiótica autista. Signo y Pensamiento. 2010; 29(57): 472-477.

8. Riviere A, Belinchon M. Reflexiones sobre el lenguaje autista. Análisis descriptivos y diferencia con la disfasia receptiva. Infancia Aprendizaje. 1981; 13: 89-120.

9. López J. Atravesando una muralla invisible: Teorías de la comunicación y semiótica autista. [Tesis de pregrado]. Bogotá, Colombia: Pontificia Universidad Javeriana, Facultad de Comunicación; 2002.

10. Colle R. Teoría Cognitiva Sistémica de la Comunicación. Santiago de Chile: Editorial San Pablo; 2002.

11. Bishop DVM. Development of the Children's Communication Checklist (CCC): A method for assessing qualitative aspects of communicative impairment in children. Journal of Child Psychology and Psychiatry. 1998; 39(6): 879-891.

12. Bishop D. Autism and specific laguage impairment: ícategorical distinction or continuum?. Autism: neural basis and treatment possibilities. Wiley, Chichester (Novartis Foundation Symposium 251). 2003: 213-234

13. Rapin I, Dunn M. Update on the language disorders of individuals on the autistic spectrum. Brain Dev. 2003; 25: 166-172.

14. Tager-Flusberg H, Joseph RM. Identifyting neurocognitive phenotypes in autism. Philos. Trans. R. Soc. B. 2003; 358: 303-314.

15. Tager-Flusberg H, Cooper J. Present and future possibilities for defining a phenotype for specific language impairment. J. Speech Lang. Hear. 1999; 42: 1275-1278.

16. Tager-Flusberg $H$. Defining language phenotypes in autism. Clin Neurosci. 2006; 6: 219-224.

17. Mulas F, Ełchepareborda M, Hernández S, Abad L, Téllez de Meneses M, Mattos L. Bases neurobiológicas de los trastornos específicos de la comunicación (espectro autista). REV NEUROL. 2005; 41 (1): 149-153.

18. Watzlawick P, Beavin J, Jackson D. Teoría de la comunicación humana. Barcelona: Editorial Herder; 1991.

19. Artigas-Pallarés J, Guitart M, GabauVila $E$. Bases genéticas de los trastornos del neurodesarrollo. Rev Neurol. 2013; 56 (1): 23-34. 